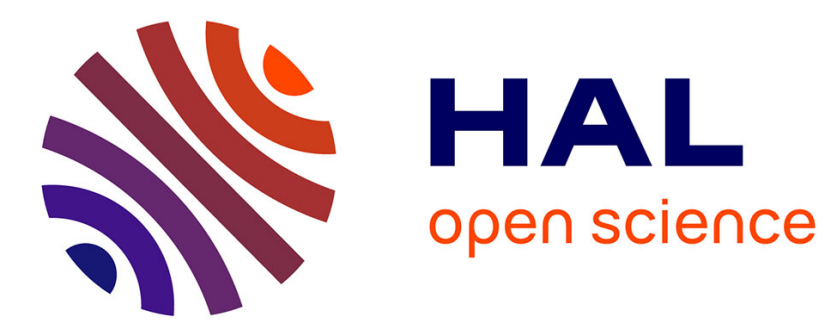

\title{
Distance between vertices of lattice polytopes
}

\author{
Anna Deza, Antoine Deza, Zhongyan Guan, Lionel Pournin
}

\section{To cite this version:}

Anna Deza, Antoine Deza, Zhongyan Guan, Lionel Pournin. Distance between vertices of lattice polytopes. Optimization Letters, 2020, 14 (2), pp.309-326. 10.1007/s11590-018-1338-7 . hal-03412184

\section{HAL Id: hal-03412184 \\ https://hal.science/hal-03412184}

Submitted on 2 Nov 2021

HAL is a multi-disciplinary open access archive for the deposit and dissemination of scientific research documents, whether they are published or not. The documents may come from teaching and research institutions in France or abroad, or from public or private research centers.
L'archive ouverte pluridisciplinaire HAL, est destinée au dépôt et à la diffusion de documents scientifiques de niveau recherche, publiés ou non, émanant des établissements d'enseignement et de recherche français ou étrangers, des laboratoires publics ou privés. 


\title{
Distance between vertices of lattice polytopes
}

\author{
Anna Deza · Antoine Deza \\ Zhongyan Guan · Lionel Pournin
}

\begin{abstract}
A lattice $(d, k)$-polytope is the convex hull of a set of points in dimension $d$ whose coordinates are integers ranging between 0 and $k$. We consider the largest possible distance $\delta(d, k)$ between two vertices in the edge-graph of a lattice $(d, k)$-polytope. We show that $\delta(5,3)$ and $\delta(3,6)$ are equal to 10 . This substantiates the conjecture whereby $\delta(d, k)$ is achieved by a Minkowski sum of lattice vectors.
\end{abstract}

Keywords Lattice Polytope · Diameter · Minkowski Sum

\section{Introduction}

Bounding the maximal possible diameter of the edge-graph of a polyhedron as a function of its dimension $d$ and the number $n$ of its facets is not only a natural question of extremal discrete geometry, but it is also historically connected with the theory of simplex methods. Larman [15] gave an upper bound on this quantity that is linear as a function of $n$, but exponential as a function of $d$, which was subsequently refined by Barnette [3] and generalized by Eisenbrand, Hähnle, Razborov, and Rothvoß [9] and Labbé, Manneville, and Santos [14]. Kalai and Kleitman [11] found an upper bound that is quasi-polynomial as a function of $d$ and $n$, which was subsequently refined by Todd [20] and Sukegawa [18]. Lower bounds have also been obtained by Klee and Walkup [12] and by Santos [17], disproving the Hirsch conjecture for unbounded polyhedra and for polytopes, respectively.

In the case of a lattice polytope, i.e. the convex hull of a set of points with integer coordinates, the range for the coordinates of the vertices can be used as an alternative to $n$. A lattice $(d, k)$-polytope is the convex hull of a set of points in dimension $d$ whose coordinates are integers ranging between 0 and $k$. Let $\delta(d, k)$ denote the largest possible diameter of a lattice $(d, k)$-polytope. The case when $k=1$ was investigated by Naddef [16] who showed that $\delta(d, 1)=d$, and thus that lattice $(d, 1)$-polytopes satisfy the Hirsch bound. This result was generalized to $\delta(d, k) \leq k d$ by Kleinschmidt and Onn [13]. The case

Anna Deza · Antoine Deza · Zhongyan Guan · Lionel Pournin

University of Toronto, Toronto, Ontario, Canada, E-mail: anna.deza@mail.utoronto.ca

McMaster University, Hamilton, Ontario, Canada, E-mail: deza@mcmaster.ca

McMaster University, Hamilton, Ontario, Canada, E-mail: guanz@mcmaster.ca

Université Paris 13, Villetaneuse, France, E-mail: lionel.pournin@univ-paris13.fr 
when $d=2$ was studied independently by Thiele [19], Balog and Bárány [2], and Acketa and Žunić [1]. It can also be found in Ziegler's book [21] as Exercise 4.15. In particular, $\delta(2, k)$ is known for all $k$. Kleinschmidt and Onn's upper bound was strengthened for $k \geq 2$ to $\delta(d, k) \leq k d-\lceil d / 2\rceil$ by Del Pia and Michini [5] with equality for $k=2$, and then to $\delta(d, k) \leq k d-\lceil 2 d / 3\rceil-(k-3)$ when $k \geq 3$ by Deza and Pournin [7]. The quantities $\delta(d, 2)=\lfloor 3 d / 2\rfloor, \delta(4,3)=8, \delta(3,4)=7$, and $\delta(3,5)=9$ were determined in [4, 5, 7]. Investigating the lower bound, Deza, Manoussakis, and Onn [6] built lattice $(d, k)$-polytopes of diameter $\lfloor(k+1) d / 2\rfloor$. These polytopes are Minkowski sums of sets of the shortest possible lattice vectors, no two of which are collinear. In this paper, we investigate Conjecture 1 stating that $\delta(d, k)$ is achieved by such polytopes.

Conjecture 1 ([6]) For any $d$ and $k, \delta(d, k)$ is achieved, up to translation, by a Minkowski sum of lattice vectors. In particular, when $k<2 d, \delta(d, k)=\lfloor(k+1) d / 2\rfloor$.

Our main contribution is the determination of $\delta(5,3)$ and $\delta(3,6)$, reported in bold in Table 1 along with the other known values of $\delta(d, k)$. The determination of $\delta(5,3)$ and $\delta(3,6)$ is detailed in Section 4

Theorem $1 \delta(5,3)$ and $\delta(3,6)$ are equal to 10.

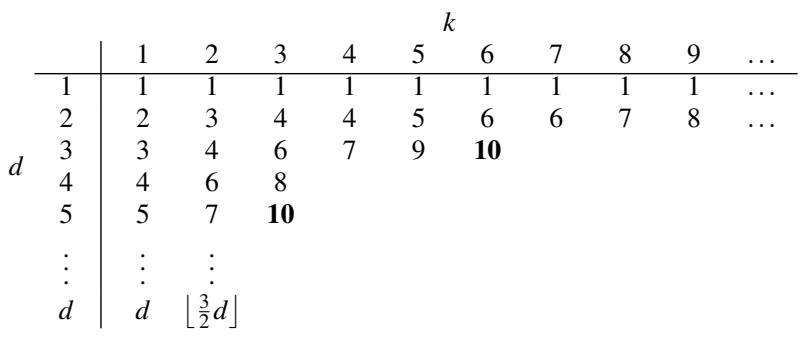

Table 1 The largest possible diameter $\delta(d, k)$ of a lattice $(d, k)$-polytope

The paper is organized as follows. Structural properties of lattice polytopes with large diameter are presented in Section 2 Those properties are used in Section 3 to generalize the computational framework introduced in [4] to determine smaller instances, allowing to prove Theorem 1 in Section 4. This manuscript is dedicated to the memory of Michel Deza who worked on a related question: bounding the diameter of a polytope in terms of the lattice points it contains, see [8].

\section{Structural Properties of Lattice Polytopes with Large Diameter}

Given two vertices $u$ and $v$ of a polytope $P$, we call $d(u, v)$ their distance in the edge-graph of $P$. If $F$ is a face of $P$, we further call $d(u, F)=\min \{d(u, v): v \in F\}$. The diameter of the edge-graph of $P$ is denoted by $\delta(P)$. The coordinates of a vector $x \in \mathbb{R}^{d}$ are denoted by $x_{1}$ to $x_{d}$, and its scalar product with a vector $y \in \mathbb{R}^{d}$ by $x \cdot y$. We recall Lemma 1 introduced by Del Pia and Michini.

Lemma 1 ([5]) Consider a lattice (d,k)-polytope $P$. If $u$ is a vertex of $P$ and $c \in \mathbb{R}^{d}$ is a vector with integer coordinates, then $d(u, F) \leq c \cdot u-\gamma$ where $\gamma=\min \{c \cdot x: x \in P\}$ and $F=\{x \in P: c \cdot x=\gamma\}$. 
We consider the following $2 d$ faces of $P$ which are key objects in our computational framework. Let $\gamma_{i}^{-}(P)=\min \left\{x_{i}: x \in P\right\}$ and $F_{i}^{-}(P)=\left\{x \in P: x_{i}=\gamma_{i}^{-}(P)\right\}$. Similarly, let $\gamma_{i}^{+}(P)=\max \left\{x_{i}: x \in P\right\}$ and $F_{i}^{+}(P)=\left\{x \in P: x_{i}=\gamma_{i}^{+}(P)\right\}$. When there is no ambiguity, $F_{i}^{-}(P)$, and $F_{i}^{+}(P)$ will be simply denoted by $F_{i}^{-}$and $F_{i}^{+}$. Considering paths from $u$ to $v$ going though $F_{i}^{-}(P)$ or $F_{i}^{+}(P)$, yields:

$$
d(u, v) \leq \min _{i=1, \ldots, d} \min \left\{\delta\left(F_{i}^{-}\right)+d\left(u, F_{i}^{-}\right)+d\left(v, F_{i}^{-}\right), \delta\left(F_{i}^{+}\right)+d\left(u, F_{i}^{+}\right)+d\left(v, F_{i}^{+}\right)\right\} .
$$

Using inequality (1) and setting $c$ as a basis vector, or its opposite, in Lemma 1 give Corollary 1. which is the key ingredient to show by induction that $\delta(d, k) \leq k d$.

Corollary 1 Let $u$ and $v$ be two vertices of a lattice $(d, k)$-polytope, then

$$
d(u, v) \leq \min _{i=1, \ldots, d} \min \left\{\delta\left(F_{i}^{-}\right)+u_{i}+v_{i}, \delta\left(F_{i}^{+}\right)+2 k-u_{i}-v_{i}\right\} .
$$

Proposition 1 is borrowed from [10], see Corollary 12.2 and Proposition 12.4 therein. It is used to prove Lemma2

Proposition 1 Let $P^{1}$ and $P^{2}$ be two polytopes in $\mathbb{R}^{d}$ and $P=P^{1}+P^{2}$ their Minkowski sum. Let $v=v^{1}+v^{2}$, such that $v^{1} \in P^{1}$ and $v^{2} \in P^{2}$. Then $v$ is a vertex of $P$ if and only if $(i) v^{1}$ and $v^{2}$ are vertices of $P^{1}$ and $P^{2}$, respectively; and (ii) there exists an objective function $c \in \mathbb{R}^{d}$ that is uniquely minimized at $v^{1}$ in $P^{1}$ and at $v^{2}$ in $P^{2}$. Moreover, if $u$ and $v$ are adjacent vertices of $P$ with Minkowski decompositions $u=u^{1}+u^{2}$ and $v=v^{1}+v^{2}$, respectively, then $u^{i}$ and $v^{i}$ are either adjacent vertices of $P^{i}$, or they coincide, for $i=1,2$.

Lemma 2 For any lattice $(d, k)$-polytope $Q$, there exists a lattice $(d, k)$-polytope $P$ of diameter at least $\delta(Q)$ satisfying $\gamma_{i}^{-}(P)=0$ and $\gamma_{i}^{+}(P)=k$ for $i=1, \ldots, d$.

Proof Assume that, for some $i, \gamma_{i}^{+}(Q)-\gamma_{i}^{-}(Q)<k$. Up to translation, we can assume that $\gamma_{i}^{-}(Q)=0$. Consider the segment $\sigma^{i}=\operatorname{conv}\left\{0,\left(k-\gamma_{i}^{+}(Q)\right) c^{i}\right\}$ where $c^{i}$ is the point whose coordinates are all equal to 0 except for the $i$-th coordinate that is equal to 1 . By construction, $Q+\sigma^{i}$ is a lattice $(d, k)$-polytope such that $\gamma_{i}^{-}\left(Q+\sigma^{i}\right)=0$ and $\gamma_{i}^{+}\left(Q+\sigma^{i}\right)=k$. Let $u$ and $v$ be two vertices of $Q$ such that $d(u, v)=\delta(Q)$. By Proposition 1 with $c=c^{i}$, there exist two vertices $u^{\prime}$ and $v^{\prime}$ of $Q+\sigma^{i}$ obtained as the Minkowski sums of $u$ and $v$, respectively with two (possibly identical) vertices of $\sigma^{i}$. Moreover, for any path of length $l$ between $u^{\prime}$ and $v^{\prime}$ in the edge-graph of $Q+\sigma^{i}$, there exists a path of length at most $l$ between $u$ and $v$ in the edge-graph of $Q$. Consequently, the distance of $u$ and $v$ in $Q$ is at most the distance of $u^{\prime}$ and $v^{\prime}$ in $Q+\sigma^{i}$. Thus, $\delta(Q) \leq \delta\left(Q+\sigma^{i}\right)$. If $\gamma_{j}^{+}\left(Q+\sigma^{i}\right)-\gamma_{j}^{-}\left(Q+\sigma^{i}\right)<k$ for some $j \neq i$, the above procedure can be repeated until no such coordinate remains.

Lemma 3 Assume that $\delta(d, k)=\delta(d-1, k)+k-g$ for an integer $g$ with $0 \leq g \leq k$.

(i) If $u$ and $v$ are two vertices of a lattice $(d, k)$-polytope such that $d(u, v)=\delta(d, k)$, then $\left|u_{i}+v_{i}-k\right| \leq$ for $i=1, \ldots, d$.

(ii) There exists a lattice $(d, k)$-polytope $P$ of diameter $\delta(d, k)$ such that the intersection of $P$ with each facet of the hypercube $[0, k]^{d}$ is, up to an affine transformation, a lattice $(d-1, k)$-polytope of diameter at least $\delta(d-1, k)-2 g$.

Proof Setting $d(u, v)=\delta(d-1, k)+k-g$ in Corollary 1 yields:

$$
\begin{aligned}
& \delta(d-1, k)+k-g \leq \delta\left(F_{i}^{-}\right)+\left(u_{i}+v_{i}\right) \text { for } i=1, \ldots, d, \\
& \delta(d-1, k)+k-g \leq \delta\left(F_{i}^{+}\right)+2 k-\left(u_{i}+v_{i}\right) \text { for } i=1, \ldots, d \text {. }
\end{aligned}
$$


Thus,

$$
\begin{aligned}
& k-g \leq u_{i}+v_{i}+\delta\left(F_{i}^{-}\right)-\delta(d-1, k) \text { for } i=1, \ldots, d, \\
& k+g \geq u_{i}+v_{i}+\delta(d-1, k)-\delta\left(F_{i}^{+}\right) \text {for } i=1, \ldots, d .
\end{aligned}
$$

Hence, since both $\delta\left(F_{i}^{-}\right)$and $\delta\left(F_{i}^{+}\right)$are at most $\delta(d-1, k)$, the inequality $k-g \leq u_{i}+v_{i} \leq$ $k+g$ holds for $i=1, \ldots, d$; that is, item $(i)$ holds. By Lemma 2 there exists a lattice $(d, k)$ polytope $P$ of diameter $\delta(d-1, k)+k-g$ such that the intersection of $P$ with each facet of the hypercube $[0, k]^{d}$ is nonempty. Let $u$ and $v$ be two vertices of $P$ such that $d(u, v)=\delta(P)$. Inequalities (4) and (5) can be rewritten as:

$$
\begin{aligned}
& \delta\left(F_{i}^{-}\right) \geq \delta(d-1, k)-g+k-\left(u_{i}+v_{i}\right) \text { for } i=1, \ldots, d, \\
& \delta\left(F_{i}^{+}\right) \geq \delta(d-1, k)-g-k+\left(u_{i}+v_{i}\right) \text { for } i=1, \ldots, d .
\end{aligned}
$$

Thus, since $k-g \leq u_{i}+v_{i} \leq k+g$ for $i=1, \ldots, d$ by item $(i), \delta\left(F_{i}^{-}\right)$and $\delta\left(F_{i}^{+}\right)$are at least $\delta(d-1, k)-2 g$ for $i=1, \ldots, d$; that is, item (ii) holds.

We recall that the bounds obtained by Del Pia and Michini [5] and Deza and Pournin [7] hold in general for lattice polytopes inscribed in rectangular boxes.

Corollary 2 (Remark 4.1 in [7]) Let $\delta\left(k_{1}, \ldots, k_{d}\right)$ denote the largest possible diameter of a polytope whose vertices have their $i$-th coordinate in $\left\{0, \ldots, k_{i}\right\}$ for $i=1, \ldots, d$ and, up to relabeling, $k_{1} \leq k_{2} \leq \cdots \leq k_{d}$. The following inequalities hold:

(i) $\delta\left(k_{1}, \ldots, k_{d}\right) \leq k_{2}+k_{3}+\cdots+k_{d}-\lceil d / 2\rceil+2$ when $k_{1} \geq 2$,

(ii) $\delta\left(k_{1}, \ldots, k_{d}\right) \leq k_{2}+k_{3}+\cdots+k_{d}-\lceil 2 d / 3\rceil+3$ when $k_{1} \geq 3$.

Observe that the statement of Remark 4.1 in [7] contains a typographical incorrectness as $k_{1}$ and $k_{d}$ were interchanged in $(i)$ and in $(i i)$. Conjecture 1 can also be stated for lattice polytopes inscribed in rectangular boxes; that is, $\delta\left(k_{1}, \ldots, k_{d}\right)$ is at most $\left\lfloor\left(k_{1}+k_{2}+\cdots+\right.\right.$ $\left.\left.k_{d}+d\right) / 2\right\rfloor$, and is achieved, up to translation, by a Minkowski sum of lattice vectors. Note that this generalization of Conjecture 1 holds for $d=2$ and for $\left(k_{1}, k_{2}, k_{3}\right)=(2,2,3)$ and $(2,3,3)$. Moreover, $\delta\left(k_{1}, k_{2}\right)=\delta\left(k_{1}, k_{1}\right)$, and $\delta(2,2,3)=\delta(2,3,3)=5$.

\section{Computational Determination of $\delta(d, k)$}

\subsection{Computational framework}

The computational framework introduced in [4] can only determine whether $\delta(d, k)$ is equal to $\delta(d-1, k)+k$. In the terms of Lemma 3 this amounts to assume that $g=0$. This case is significantly easier than when $g>0$ since it can then be assumed that both $\delta\left(F_{i}^{-}\right)$and $\delta\left(F_{i}^{+}\right)$are equal to $\delta(d-1, k)$ and that the vertices $u$ and $v$ such that $d(u, v)=\delta(d, k)$ satisfy $u_{i}+v_{i}=k$ for $i=1, \ldots, d$. In addition, the computations were performed for $d=3$; that is, for instances such that the determination of all lattice $(d-1, k)$-polytopes of diameter $\delta(d-1, k)$ is computationally inexpensive compared to higher dimensions. To handle the case $g>0$ and be able to determine all lattice $(d-1, k)$-polytopes of diameter $\delta(d-1, k)$ for $d>3$, we introduce an enhanced algorithm exploiting the structural properties presented in Section 2. We are able to recompute previously determined values of $\delta(d, k)$ in a few seconds and obtain previously intractable values. In addition, the enhanced algorithm can be used to generate all the lattice $(d, k)$-polytopes maximizing the diameter. The enhanced algorithm is presented in Section 3.2 and illustrated for small instances of $(d, k)$. 
The determination of $\delta(d, k)$ is performed via the determination of $g(d, k)=\delta(d-$ $1, k)+k-\delta(d, k)$. For example, $g(d, 1)=0$ for $d \geq 2, g(d, 2)=d \bmod 2$ for $d \geq 2$, and $g(3,3)=1$. Assuming that $g(d-1, k)$ is known, the determination of $g(d, k)$ requires to first determine all the lattice $(d-1, k)$-polytopes achieving a diameter of $\delta(d-1, k)$. The algorithm implicitly enumerates all possible lattice $(d, k)$-polytopes $P$ of diameter at least some target value. The search space is reduced by exploiting integrality and convexity. In particular, a divide and conquer strategy is adopted by implicitly enumerating all pairs $(u, v)$ of vertices such that $d(u, v)=\delta(P)$. The algorithm crucially exploits the knowledge about all lattice $(d-1, k)$-polytopes with sufficiently large diameters, and the symmetries of the hypercube. The construction consists of two steps: the shelling step and the inner step. In the shelling step, for a given pair $(u, v)$, the algorithm tries to embed lattice $(d-1, k)$-polytopes with sufficiently large diameters onto the $2 d$ intersections of $P$ with the facets of the hypercube $[0, k]^{d}$. These embeddings must be consistent; that is, given two embeddings $E_{1}$ and $E_{2}$, the intersection of $E_{1}$ with the facet of $[0, k]^{d}$ containing $E_{2}$ should be equal to the intersection of $E_{2}$ with the facet of $[0, k]^{d}$ containing $E_{1}$. The algorithm also upper bounds the distance of $u$ and $v$ from each intersection of $P$ with the facets of the hypercube $[0, k]^{d}$, and it aborts if it detects $(i)$ a shortcut between $u$ and $v,(i i)$ that $u$ or $v$ become not extremal, or $(i i i)$ that no shelling would achieve the target diameter. If none of the stopping criteria is met, the shelling step returns a list of shellings; that is, a list of choices for the $2 d$ intersections of $P$ with the facets of the hypercube $[0, k]^{d}$, for which it might be possible to construct a lattice $(d, k)$-polytope achieving the target diameter. Consequently, for each obtained shelling, the inner step is performed where all possible points in $\{1,2, \ldots, k-1\}^{d}$ are considered to be added as vertices of $P$. Finally, the lattice $(d, k)$-polytopes with an empty intersection with at least one facet of $[0, k]^{d}$ are derived from the current output to complete the enumeration. If the output is empty we can conclude that no lattice $(d, k)$-polytope with the target diameter exists; the target diameter is lowered by one, and so forth. If the output is nonempty, we obtain all lattice $(d, k)$-polytopes with the target diameter and the algorithm can run again for $d+1$. In the remainder of this section, a more detailed description is given.

\subsection{Algorithm to determine whether $\delta(d, k)=\delta(d-1, k)+k-g$}

Assuming that the value for $\delta(d-1, k)$ is known, the initial lower bound for $g(d, k)$ is $g=0$; that is, the initial upper bound used for $\delta(d, k)$ is $\delta(d-1, k)+k$. Using the necessary conditions derived from the structural properties presented in Lemma 2 the algorithm checks whether there exists a lattice $(d, k)$-polytope of diameter $\delta(d-1, k)+k$. If no such polytope exists, $g$ is update to 1 ; that is, the upper bound is updated to $\delta(d-1, k)+k-1$, and the computational framework checks whether there exists a lattice $(d, k)$-polytope of diameter $\delta(d-1, k)+k-1$, and so on. The lower bound for $\delta(d, k)$, and thus the corresponding upper bound for $g(d, k)$, is provided by the Minkowski sum of primitive lattice vectors proposed by Deza, Manoussakis, and Onn [6]. For instance, the initial upper bound for $(d, k)=(3,6)$ is $\delta(3,6) \leq \delta(2,6)+6=12$ while the lower bound is $\delta(3,6) \geq 10$; that is, $0 \leq g(3,6) \leq 2$. The algorithm first assumes that $\delta(3,6)$ is equal to 12 and determines that no lattice $(3,6)$ polytope has diameter 12 ; that is, $g(3,6) \geq 1$. Then, assuming that $\delta(3,6)$ is equal to 11 , the algorithm determines that no lattice $(3,6)$-polytope has diameter 11 ; that is, $g(3,6) \geq 2$. Thus, we can conclude that $\delta(3,6)$ is equal to 10 ; that is, $g(3,6)$ is equal to 2 . 
3.2.1 Input and output

The input of the algorithm consists of a triple $(d, k, g)$ where:

(i) $d$ is the dimension,

(ii) $k$ is the largest possible entry of a coordinate of a vertex; that is, $x_{i} \in\{0,1, \ldots, k\}$,

(iii) $g$ is a parameter determining the diameter we wish to rule out (or achieve); i.e. we assume that the diameter is at least $\delta(d-1, k)+k-g$.

The output consists of, up to the symmetries of the hypercube $[0, k]^{d}$, all the lattice $(d, k)$ polytopes of diameter at least $\delta(d-1, k)+k-g$. An empty output provides a certificate that $\delta(d, k)<\delta(d-1, k)+k-g$, and a nonempty output shows that $\delta(d, k) \geq \delta(d-1, k)+$ $k-g$. Note that the algorithm requires the determination of all lattice $(d-1, k)$-polytopes of diameter $\delta(d-1, k)$. For $d=3$, the enumeration of all lattice $(2, k)$-polygons of diameter $\delta(2, k)$ is straightforward and inexpensive compared to the overall computational cost. For $d \geq 4$, the enumeration of all, up to the symmetries of $[0, k]^{d}$, lattice $(d-1, k)$-polytopes of diameter $\delta(d-1, k)$ is obtained as an output of the algorithm run with the input $(d-1, k, g)$ where $\delta(d-1, k)=\delta(d-2, k)+k-g$ assuming that all lattice $(d-2, k)$-polytopes of the required diameter are known.

For example, in order to determine whether $\delta(4,3)=9$ we need to run the algorithm with $(d, k, g)=(4,3,0)$. Thus, we need to enumerate all lattice $(3,3)$-polytopes of diameter 6; that is, we need to run the algorithm with $(d, k, g)=(3,3,1)$. The algorithm output the 9 , up to the symmetries of $[0,3]^{3}$, lattice $(3,3)$-polytopes of diameter 6 . Then, running the algorithm with $(d, k, g)=(4,3,0)$ amounts to finding a consistent combination of embeddings of lattice $(3,3)$-polytopes of diameter 6 onto the 8 facets of the hypercube $[0,3]^{4}$. As any such obtained lattice $(4,3)$-polytope is of diameter at most 8 , the output is the empty set, and thus $\delta(4,3)<9$. Similarly, if the enumeration of all lattice $(d-1, k)$ polytopes of diameter at least $\delta(d-1, k)-1$ is required, the list, up to the symmetries of $[0, k]^{d-1}$, is obtained as an output of the algorithm run with the input $(d-2, k, g)$ where $\delta(d-1, k)-1=\delta(d-2, k)+k-g$.

3.2.2 Generating all potential pairs $(u, v)$ of vertices of a lattice $(d, k)$-polytope such that $d(u, v)=\delta(d-1, k)+k-g$

A critical ingredient of the method consists in reducing, as much as possible, the number of pairs $\{u, v\}$ that must be considered. This is achieved by noticing that some restricting conditions can be assumed without loss of generality.

First, by the symmetries of the hypercube $[0, k]^{d}$, we can assume that the coordinates of $u$ satisfy:

$$
u_{i} \leq u_{i+1} \leq\lfloor k / 2\rfloor \text { for } i=1, \ldots, d-1
$$

In addition, by item $(i)$ of Lemma 3 , we can assume that the coordinates of $u$ and $v$ satisfy:

$$
k-g \leq u_{i}+v_{i} \leq k+g \text { for } i=1, \ldots, d .
$$

Further, by the symmetries of the hypercube $[0, k]^{d}$ acting on the pair $\{u, v\}$ and assuming that all $u$ are generated in the lexicographic order (denoted by $\prec$ in the following), we can 
assume that the coordinates of $u$ and $v$ satisfy the following conditions where $\tilde{v}$ is the point consisting of the coordinates of $v$ reordered lexicographically:

$$
\begin{aligned}
\left\{v_{i} \leq v_{i+1} \text { if } u_{i}=u_{i+1}\right\} & \text { for } i=1, \ldots, d-1, \\
\tilde{v} \prec(k, \ldots, k)-u & \text { if }\left\{v_{i} \geq\lceil k / 2\rceil \text { for } i=1, \ldots, d\right\}
\end{aligned}
$$

Finally, we can exploit the fact that the intersections with the facets of $[0, k]^{d}$ must be of sufficiently large diameter. Let $\mathscr{V}_{d, k, g}$ denote the set formed by all the vertices of all the lattice $(d, k)$-polytopes of diameter at least $\delta(d, k)-g$. Let $\bar{v}_{i}$ denote the point in $\mathbb{R}^{d-1}$ consisting of all coordinates of $v$ except $v_{i}$, and let $g_{i}^{-}=g+u_{i}+v_{i}-k$ and $g_{i}^{+}=g+k-\left(u_{i}+v_{i}\right)$. The following conditions are necessary for $u$ and $v$ to be vertices of a lattice $(d, k)$-polytope such that $d(u, v)$ is at least $\delta(d-1, k)+k-g$ :

$$
\begin{aligned}
\left\{\bar{u}_{i} \in \mathscr{V}_{d-1, k, g_{i}^{-}} \text {if } u_{i}=0\right\} & \text { for } i=1, \ldots, d, \\
\left\{\bar{v}_{i} \in \mathscr{V}_{d-1, k, g_{i}^{+}} \text {if } v_{i}=k\right\} & \text { for } i=1, \ldots, d
\end{aligned}
$$

Let $\mathscr{P}_{d, k, g}$ denote the set of all the points with integer coordinates that belong to the intersection of all the lattice $(d, k)$-polytopes of diameter at least $\delta(d, k)-g$. Let $\mathscr{C}_{d, k, g}^{u, v}$ denote the convex hull of $u, v$, and the following set of points:

$$
\left[\bigcup_{i=1}^{d}\left\{x \in \mathbb{R}^{d}: x_{i}=0 \text { and } \bar{x}_{i} \in \mathscr{P}_{d-1, k, g_{i}^{-}}\right\}\right] \cup\left[\bigcup_{i=1}^{d}\left\{x \in \mathbb{R}^{d}: x_{i}=k \text { and } \bar{x}_{i} \in \mathscr{P}_{d-1, k, g_{i}^{+}}\right\}\right]
$$

The following condition is necessary for $u$ and $v$ to be vertices of a lattice $(d, k)$-polytope such that $d(u, v)$ is at least $\delta(d-1, k)+k-g$ :

$$
u \text { and } v \text { are vertices of } \mathscr{C}_{d, k, g}^{u, v} \text {. }
$$

Let us illustrate the conditions that can be assumed for the pair $\{u, v\}$ by considering the case $(d, k, g)=(3,6,0)$. In other words, we assume that $u$ and $v$ are vertices of a lattice $(3,6)$-polytope of diameter 12 . Since $g=0$, we can assume that:

$$
\begin{gathered}
u_{1} \leq u_{2} \leq u_{3} \leq 3, \\
u+v=(6,6,6), \\
\left\{\bar{u}_{i} \in \mathscr{V}_{2,6,0} \text { if } u_{i}=0\right\} \quad \text { for } i=1,2,3, \\
u \text { is a vertex of } \mathscr{C}_{3,6,0}^{u, v} \text { if } u_{1} \neq 0 .
\end{gathered}
$$

Among the 20 points $u$ satisfying $u_{1} \leq u_{2} \leq u_{3} \leq 3$, the only ones such that $u_{1}=0$ and $\left(u_{2}, u_{3}\right) \in \mathscr{V}_{2,6,0}$ are $(0,0,1),(0,0,2),(0,0,3),(0,1,1)$, and $(0,1,2)$. In addition, no point such that $u_{1} \neq 0$ is a vertex of $\mathscr{C}_{3,6,0}^{u, v}$. Thus, since $u+v=(6,6,6)$, we need to consider only 5 pairs of vertices $\{u, v\}$. The sets $\mathscr{V}_{2,6,0}$ and $\mathscr{P}_{2,6,0}$ can be easily computed and are illustrated in Figure 1 . Note that both $\mathscr{V}_{d, k, g}$ and $\mathscr{P}_{d, k, g}$ are invariant under the symmetries of $[0, k]^{d}$. 

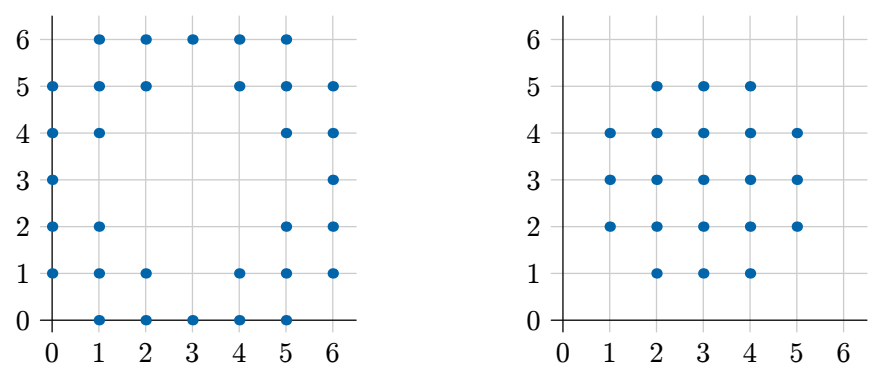

Fig. 1 The sets $\mathscr{V}_{2,6,0}$ and $\mathscr{P}_{2,6,0}$

3.2.3 Main subroutine to determine whether there exists a lattice $(d, k)$-polytope with vertices $u$ and $v$ such that $d(u, v)=\delta(d-1, k)+k-g$

For each pair $\{u, v\}$ generated by the steps described in Section 3.2.2, we run the main subroutine to determine whether there exists a lattice $(d, k)$-polytope with vertices $u$ and $v$ such that $d(u, v)=\delta(d-1, k)+k-g$. Such polytopes are generated by considering all possible choices for the $2 d$ intersections with the facets of the hypercube $[0, k]^{d}$. This step is called the shelling step. The order in which the intersections are considered is critical to reduce the search space. It is equally critical to identify, as early as possible, paths between $u$ and $v$ possibly induced by the shelling process. A set of choices for the $2 d$ intersections with the facets of the hypercube $[0, k]^{d}$ obtained by the shelling step is called a shelling. The output of the shelling step consists in the list of all the shellings generated by performing the shelling step for all possible pairs $\{u, v\}$. Shellings that are duplicate, up to the symmetries of the hypercube $[0, k]^{d}$, are removed. If the output of the shelling step is empty, the process stops and we can conclude that $\delta(d, k)<\delta(d-1, k)+k-g$.

If the output of the shelling step is nonempty, all possible points with integer coordinates ranging from 1 to $k-1$ are considered as potential additional vertices for each generated shelling. This step is called the inner step. Again, structural properties are critical to prune the search space. The output of the inner step consists in the list of all the generated lattice $(d, k)$-polytopes after duplicates, up to the symmetries of the hypercube $[0, k]^{d}$, and lattice $(d, k)$-polytopes of diameter at most $\delta(d-1, k)+k-g-1$ have been removed. If the output of the inner step is empty, we can conclude that $\delta(d, k)<\delta(d-1, k)+k-g$.

3.2.4 Two certificates that no lattice $(d, k)$-polytopes with vertices $u$ and $v$ such that $d(u, v)=\delta(d-1, k)+k-g$ exist

Let $\Gamma$ denote the graph defined by the currently known edges and vertices of a lattice $(d, k)$ polytope. $\Gamma$ is initially set to $\{u, v\}$. Let $d_{\Gamma}(x, y)$ denote the distance in $\Gamma$ between two vertices $x$ and $y$. We consider the following upper bounds for the distance between $u$ or $v$ 
and the intersection with a facet of the $[0, k]^{d}$, and two upper bounds for $d(u, v)$.

$$
\begin{array}{rr}
\widetilde{d}\left(u, F_{i}^{-}\right)=\min _{w \in \Gamma}\left\{d_{\Gamma}(u, w)+w_{i}\right\} & \text { for } i=1, \ldots, d, \\
\widetilde{d}\left(u, F_{i}^{+}\right)=\min _{w \in \Gamma}\left\{d_{\Gamma}(u, w)+k-w_{i}\right\} & \text { for } i=1, \ldots, d, \\
\widetilde{d}\left(v, F_{i}^{-}\right)=\min _{w \in \Gamma}\left\{d_{\Gamma}(v, w)+w_{i}\right\} & \text { for } i=1, \ldots, d, \\
\widetilde{d}\left(v, F_{i}^{+}\right)=\min _{w \in \Gamma}\left\{d_{\Gamma}(v, w)+k-w_{i}\right\} & \text { for } i=1, \ldots, d .
\end{array}
$$

Note that setting $w$ to $u$ or $v$ gives $\widetilde{d}\left(u, F_{i}^{-}\right) \leq u_{i}, \widetilde{d}\left(v, F_{i}^{-}\right) \leq v_{i}, \widetilde{d}\left(u, F_{i}^{+}\right) \leq k-u_{i}$, and $\widetilde{d}\left(v, F_{i}^{+}\right) \leq k-v_{i}$. The following quantity $d_{\circ}(u, v)$, where both $\delta\left(F_{i}^{-}\right)$and $\delta\left(F_{i}^{+}\right)$are bounded from above by $\delta(d-1, k)$, is an upper bound for $d(u, v)$ by inequality $[1]$ :

$$
d_{\circ}(u, v)=\min _{i=1, \ldots, d}\left\{\min \left\{\widetilde{d}\left(u, F_{i}^{-}\right)+\widetilde{d}\left(v, F_{i}^{-}\right)+\delta\left(F_{i}^{-}\right), \widetilde{d}\left(u, F_{i}^{+}\right)+\widetilde{d}\left(v, F_{i}^{+}\right)+\delta\left(F_{i}^{+}\right)\right\}\right\} .
$$

Each time a choice for the intersection with a facet of the hypercube $[0, k]^{d}$ is considered, the value of $d_{\circ}(u, v)$ is updated. Similarly, since $\Gamma$ is a subgraph of the edge-graph, $d_{\Gamma}(u, v)$ is another upper bound for $d(u, v)$. Thus, we consider the following nonnegative parameter $\gamma$ defined as:

$$
\gamma=\delta(d-1, k)+k-g-\min \left\{d_{\Gamma}(u, v), d_{\circ}(u, v)\right\} .
$$

Consequently, $\gamma>0$ is a certificate that there does not exist a lattice $(d, k)$-polytope with vertices $u$ and $v$ such that $d(u, v)=\delta(d-1, k)+k-g$.

Another estimate that is updated along with $\Gamma$ is the convex hull $\mathscr{C}_{d, k, g}^{\Gamma}$ of the vertex set of $\Gamma$ and the following set of points:

$$
\left[\bigcup_{i=1}^{d}\left\{x \in \mathbb{R}^{d}: x_{i}=0 \text { and } \bar{x}_{i} \in \mathscr{P}_{d-1, k, g_{i}^{-}}\right\}\right] \cup\left[\bigcup_{i=1}^{d}\left\{x \in \mathbb{R}^{d}: x_{i}=k \text { and } \bar{x}_{i} \in \mathscr{P}_{d-1, k, g_{i}^{+}}\right\}\right] .
$$

The following condition is a certificate that there does not exist a lattice $(d, k)$-polytope with vertices $u$ and $v$ such that $d(u, v)=\delta(d-1, k)+k-g$ :

$$
u \text { or } v \text { is not a vertex of } \mathscr{C}_{d, k, g}^{\Gamma} \text {. }
$$

\subsubsection{Shelling step}

Given a triple $(d, k, g)$ and a pair $\{u, v\}$, the scores of $F_{i}^{-}$and $F_{i}^{+}$are $g_{i}^{-}=g+u_{i}+v_{i}-k$ and $g_{i}^{+}=g+k-\left(u_{i}+v_{i}\right)$, respectively. The intersections with the facets of $[0, k]^{d}$ are ordered by their score, in a non-decreasing order. If two intersections or more have the same score, the number of currently known vertices of $P$ belonging to the intersection is used as a tiebreaker, starting with the one containing the largest number of such vertices. As a secondary tie-breaker, an intersection containing $u$ or $v$ is considered before one containing neither, with "containing $u$ " given priority over "containing $v$ " as a further tie-breaker. If none of those rules apply, the default order is $F_{1}^{-}, \ldots, F_{d}^{-}, F_{1}^{+}, \ldots, F_{d}^{+}$.

Every time an intersection $F_{i}^{-}$or $F_{i}^{+}$is considered, we generate the set of lattice $(d-1, k)$ polytopes of diameter at least $\delta(d-1, k)-g_{i}^{-}$or at least $\delta(d-1, k)-g_{i}^{+}$, respectively, and having $\bar{x}_{i}$ as a vertex when $x$ is a vertex from $\Gamma$ such that $x_{i}=0$ or $x_{i}=k$, respectively. 
After one such lattice $(d-1, k)$-polytope is assigned to form, up to an affine transformation, the chosen intersection with $[0, k]^{d}$, its vertices and edges are added to $\Gamma$. Consequently, the values of $\gamma$ and $\mathscr{C}_{d, k, g}^{\Gamma}$ are updated. If $u$ or $v$ is not a vertex of $\mathscr{C}_{d, k, g}^{\Gamma}$, or if $\gamma>0$, the search can be pruned at this node. Typically, the very first chosen intersection with $[0, k]^{d}$ may yield a certificate of non-existence. Note that all scores $g_{i}^{-}$and $g_{i}^{+}$are updated, i.e. monotonically nonincreased, during the shelling process. Namely, each time a choice for the intersection with a facet of the hypercube $[0, k]^{d}$ is considered, the scores $g_{i}^{-}$and $g_{i}^{+}$of not yet considered intersections are updated, if lowered, to $g_{i}^{-}=g+\widetilde{d}\left(u, F_{i}^{-}\right)+\widetilde{d}\left(v, F_{i}^{-}\right)-k$ and to $g_{i}^{+}=g+\widetilde{d}\left(u, F_{i}^{+}\right)+\widetilde{d}\left(v, F_{i}^{+}\right)-k$.

In order to illustrate the shelling step, we first consider the case $(d, k, g)=(3,6,0)$. As discussed in Section 3.2.1 there are 5 pairs $\{u, v\}$ to consider. Since $g=0$, the score of any intersection with $[0, k]^{d}$ is zero. The only currently known vertices are $u$ and $v$ and $u_{1}=0$ for all the 5 pairs. Thus, the first considered intersection is $F_{1}^{-}$. Consequently, for each $\{u, v\}$, we generate the set of lattice $(2,6)$-polytopes of diameter 6 having $\left(u_{2}, u_{3}\right)$ as a vertex. One can easily check that $\gamma>0$ for each such choice. Thus, the shelling step terminates after considering $F_{1}^{-}$for all possible pairs $\{u, v\}$ and we can conclude that $\delta(3,6)<12$ in a matter of seconds. Another simple example is the case $(d, k, g)=(3,4,0)$ for which the output of the shelling step consists in a unique shelling where the 6 intersections with the facets of $[0,4]^{3}$ are, up to an affine transformation, the octagonal lattice $(2,3)$-polytope. See Figure 2 for an illustration where the edges of the shelling are shown in blue. As no point whose coordinates are $\{1,2,3\}$-valued can be added to this unique shelling as a potential vertex, the inner step is reduced to check whether the diameter of the convex hull associated to the unique shelling achieves $\delta(2,4)+4-0=8$. As the diameter is equal to 7 , the output of the inner step is empty. Thus, we can conclude that $\delta(3,4)<8$; that is, $\delta(3,4)=7$.

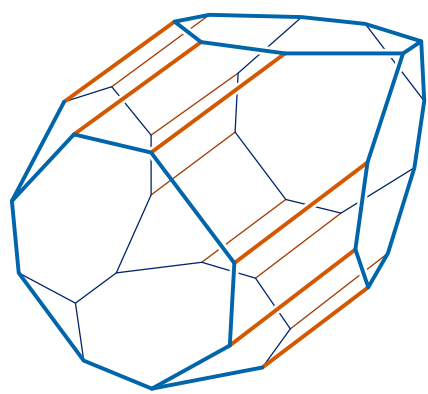

Fig. 2 The unique shelling generated for $(d, k, g)=(3,4,0)$

\subsubsection{Inner step}

The input for the inner step is the output of the shelling step, assuming it is nonempty. For each shelling, the inner step considers all the inner points $p$; that is, all the points $p$ such that $p_{i} \in\{1, \ldots, k-1\}$ for $i=1, \ldots, d$. Let $\mathscr{C}_{d, k, g}^{\Gamma \cup p}$ denote the convex hull of $p$ and $\mathscr{C}_{d, k, g}^{\Gamma}$. A necessary condition for $p$ to be a vertex of a lattice $(d, k)$-polytope of diameter 
$\delta(d-1, k)+k-g$ is:

$$
p \text { is a vertex of } \mathscr{C}_{d, k, g}^{\Gamma \cup p} \text {. }
$$

The generated lattice $(d, k)$-polytopes whose diameter is at most $\delta(d-1, k)+k-g-1$ are removed. Similarly the duplicates, up to the symmetries of the hypercube $[0, k]^{d}$, are removed. If the output of the inner step is empty, we can conclude that $\delta(d, k)$ is strictly less than $\delta(d-1, k)+k-g$. Otherwise, we can conclude that $\delta(d, k)=\delta(d-1, k)+k-g$, and the output of the inner step provides, up to the symmetries of the hypercube $[0, k]^{d}$, all lattice $(d, k)$-polytopes of diameter $\delta(d-1, k)+k-g$ whose intersection with each facet of the hypercube $[0, k]^{d}$ is nonempty. Further computations allow to determine all lattice $(d, k)$ polytopes of diameter $\delta(d-1, k)+k-g$ with an empty intersection with at least one facet of the hypercube $[0, k]^{d}$, as detailed in Section 3.3

In order to illustrate the inner step, we consider the case $(d, k, g)=(3,4,2)$ and the pair $\{u, v\}=\{(0,0,0),(4,4,4)\}$. In other words, we assume that $u=(0,0,0)$ and $v=(4,4,4)$ are vertices of a lattice $(3,4)$-polytope such that $d(u, v)=6$. Considering this pair, we first perform the shelling step. Since the scores satisfy $g_{1}^{-}=g_{2}^{-}=g_{3}^{-}=g_{1}^{+}=g_{2}^{+}=g_{3}^{+}=2$, the 6 intersections with the facets of $[0,4]^{3}$ are of diameter at least 2 . One can check that the shelling step output includes the shelling consisting of the 6 identical facets depicted in Figure 3 using blue lines. Each of these facets is, up to an affine transformation, equal to the square $[0,1]^{2}$. Out of the 27 points whose coordinates are $\{1,2,3\}$-valued, 15 are contained in the convex hull of this shelling. Thus, the inner step must consider 12 inner points as possible vertices to be added to this shelling. One can check that, up to the symmetries of $[0,4]^{3}, 214$ lattice $(3,4)$-polytopes are generated and that all of them have diameter at most 5 . There are exactly 8 polytopes among them whose diameter is equal to 5 . One of these lattice $(3,4)$-polytopes of diameter 5 is represented in Figure 3 where the 6 added vertices are show in green and the edges of the intersections with the facets of $[0,4]^{3}$ are shown in blue. Note that since polytopes of diameter at most $\delta(d-1, k)+k-g-1=4+4-2-1=5$ are removed, none of the 214 lattice $(3,4)$-polytopes generated by this shelling are part of the output of the inner step for $(d, k, g)=(3,4,2)$.

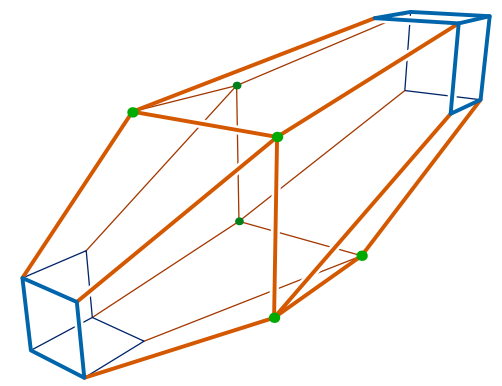

Fig. 3 A polytope considered by the inner step for $(d, k, g)=(3,4,2)$ and $\{u, v\}=\{(0,0,0),(4,4,4)\}$ 
3.3 Generation of all the lattice $(d, k)$-polytopes of diameter at least $\delta(d-1, k)+k-g$

Running the algorithm for $(d, k, g)$ allows to determine, up to the symmetries of $[0, k]^{d}$, the set of all the lattice $(d, k)$-polytopes with diameter at least $\delta(d-1, k)+k-g$ whose intersection with each facet of $[0, k]^{d}$ is nonempty. In this section we outline how the ones with an empty intersection with at least one facet of $[0, k]^{d}$ can be derived from this set.

Using the notations of Lemma 2 let $I(Q)$ denote the set of the coordinates $i$ such that $\gamma_{i}^{+}(Q)-\gamma_{i}^{-}(Q)<k$. Consider a lattice $(d, k)$-polytope $Q$ of diameter at least $\delta(d-1, k)+$ $k-g$ such that $I(Q) \neq \emptyset$. For all $i \in I(Q)$, we can assume, up to translation, that $\gamma_{i}^{-}(Q)=0$ and consider the segment $\sigma^{i}=\operatorname{conv}\left\{0,\left(k-\gamma_{i}^{+}(Q)\right) c^{i}\right\}$. Let $S$ denote the Minkowski sum of all $\sigma^{i}$ for $i \in I(Q)$. As shown in the proof of Lemma 2, $Q+S$ is a lattice $(d, k)$-polytope of diameter at least $\delta(Q)$ satisfying $I(Q+S)=\emptyset$. In other words, $Q+S$ is, up to the symmetries of $[0, k]^{d}$, in the output of the algorithm ran for $(d, k, g)$. Note that setting $P^{1}=Q$ and $P^{2}=[0, s]$ where $s_{i} \geq 0$ for all $i$ in Proposition 1 gives Remark 1

Remark 1 Consider a segment $\sigma=[0, s]$; a point $v^{\prime}$ is a vertex of $Q+\sigma$ if and only if there exists an objective function $c \in \mathbb{R}^{d}$ that is uniquely minimized at $v$ in $Q$ and $(i) v^{\prime}=v$ and $c$ is uniquely minimized at 0 in $\sigma$, or $(i i) v^{\prime}=v+s$ and $c$ is uniquely minimized at $s$ in $\sigma$. Moreover, if $u^{\prime}$ and $v^{\prime}$ are adjacent vertices of $Q+\sigma$, then either $\left(u^{\prime}, v^{\prime}\right)$ is equal to $(u, v)$ or to $(u+s, v+s)$ where $u$ and $v$ are adjacent vertices of $Q$, or it is equal to $(u, u+s)$ where $u$ is a vertex of $Q$.

Consequently, up to translation and up to the symmetries of the hypercube $[0, k]^{d}$, the set of the lattice $(d, k)$-polytopes $Q$ of diameter at least $\delta(d-1, k)+k-g$ such that $I(Q) \neq \emptyset$ can be generated as follows:

(i) for each lattice $(d, k)$-polytope $P$ in the output of the algorithm ran for $(d, k, g)$, check whether $P=Q+\sigma$ where $Q$ is a lattice $(d, k)$-polytope and $\sigma$ a lattice segment. By Remark 1 this can be done by checking whether $P$ and $P+\sigma$ have the same number of vertices,

(ii) for each $P$ such that $P=Q+\sigma$ found at step $(i)$, determine $Q$ and check whether $\delta(Q) \geq$ $\delta(d-1, k)+k-g$.

As for the shelling and inner steps, the symmetries of the hypercube $[0, k]^{d}$ are used to remove duplicates generated within steps $(i)$ and (ii). The set of lattice segments $\sigma$ considered in step $(i)$ can be limited to a few segments whose coordinates are relatively prime and used iteratively. For an illustration, we consider the case $(d, k, g)=(3,3,1)$. As discussed in Section 4.2 the output of the algorithm consists in 9 lattice $(3,3)$-polytopes of diameter 6 whose intersection with each facet of $[0,3]^{3}$ is nonempty. One can check that, in order to perform step $(i)$, it is enough to consider for $\sigma$, iteratively, the vectors $v$ such that $\|v\|_{2} \leq \sqrt{2}$. All the 9 considered lattice $(3,3)$-polytopes of diameter 6 can be written as $Q+\sigma$. Performing step (ii), one can check that $\delta(Q)=5$ for each such $Q$. Thus, there is no lattice (3,3)-polytope $Q$ of diameter 6 such that $I(Q) \neq \emptyset$.

\section{Proof of Theorem 1}

Theorem 1 is obtained by computationally verifying that the output of the inner step is empty for $(d, k, g)=(3,6,1)$ and $(5,3,0)$. Thus, $\delta(3,6)<11$ and $\delta(5,3)<11$; that is, $\delta(3,6)=\delta(5,3)=10$. Running the algorithm for $(5,3,0)$ requires the determination of all 
lattice (3,3)-polytopes of diameter 5 or 6 and all lattice (4,3)-polytopes of diameter 8 . The algorithm is implemented in $C \#$ and experiments were carried out on a MacBook Pro with a $2.8 \mathrm{GHz}$ i7 processor and $16 \mathrm{~GB}$ of RAM.

\subsection{Determination of $\delta(3,6)$}

As mentioned in Section 3.2.5 the output of the shelling step is empty for $(d, k, g)=(3,6,0)$ and thus we can conclude that $\delta(3,6)<12$. Running the algorithm for $(d, k, g)=(3,6,1)$ is computationally efficient because of two key properties.

First, there are only 4 lattice $(2,6)$-polytopes of diameter 6 , see Figure 4 for an illustration.
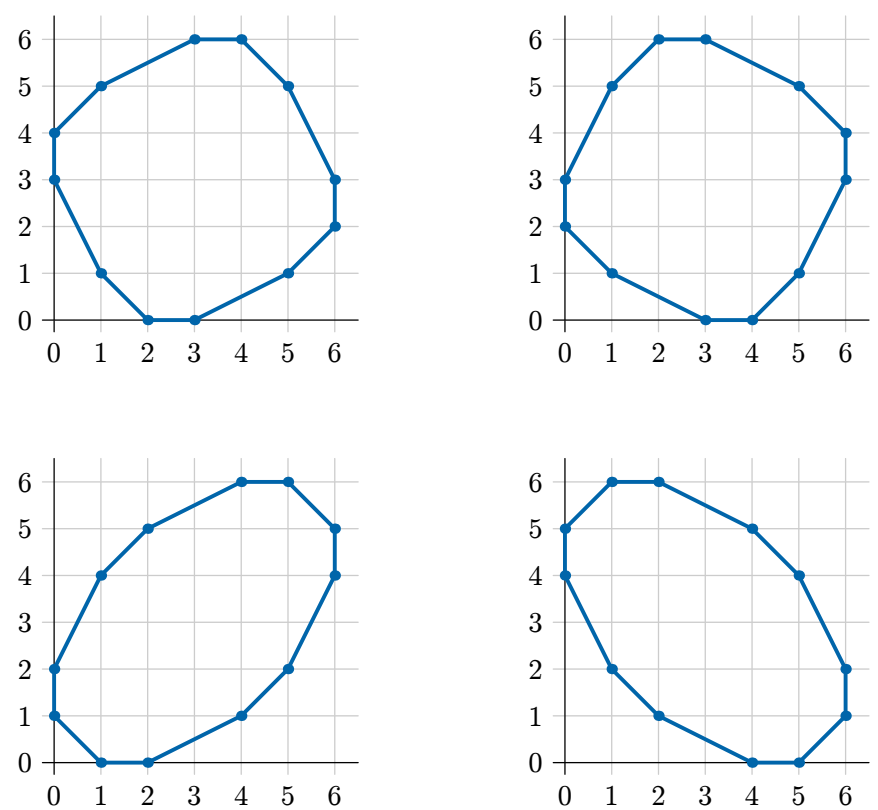

Fig. 4 All lattice (2,6)-polytopes of diameter 6

Second, for $d=2$, there are only 8 lattice vectors $v$ such that $\|v\|_{\infty} \leq 1$. Thus, any lattice $(2,6)$-polytope of diameter 5 or 6 includes at least 2 edges $e$ such that $\|e\|_{\infty} \geq 2$.

Consequently, unless both $u$ and $v$ are inner points, the update of the scores $g_{i}^{-}=g+$ $\widetilde{d}\left(u, F_{i}^{-}\right)+\widetilde{d}\left(v, F_{i}^{-}\right)-k$, respectively of $g_{i}^{+}=g+\widetilde{d}\left(u, F_{i}^{+}\right)+\widetilde{d}\left(v, F_{i}^{+}\right)-k$, implies that $g_{i}^{-}$or $g_{i}^{+}$is updated to zero for some $i$ after the first intersection with a facet of $[0,6]^{3}$ is considered in the shelling step. As $g_{i}^{-}=0$ or $g_{i}^{+}=0$ implies that $\delta\left(F_{i}^{-}\right)=6$ or $\delta\left(F_{i}^{+}\right)=6$, respectively, there are at most 4 lattice $(2,6)$-polytopes to consider for the next intersection with a facet of $[0,6]^{3}$, and so forth. As an illustration, consider the pair $\{u, v\}=\{(0,0,0),(6,6,6)\}$. Initially, the scores satisfy $g_{1}^{-}=g_{2}^{-}=g_{3}^{-}=g_{1}^{+}=g_{2}^{+}=g_{3}^{+}=1$ and the shelling step starts 


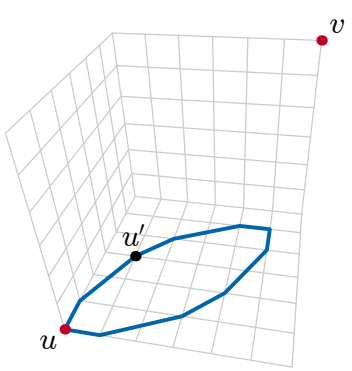

Fig. 5 Initial iteration of the shelling step for $(d, k, g)=(3,6,1)$ and $\{u, v\}=\{(0,0,0),(6,6,6)\}$

by considering a lattice $(2,6)$-polytope of diameter at least 5 for $F_{1}^{-}$. For example, assume that $F_{1}^{-}$is, up to an affine transformation, the lattice $(2,5)$-polytope obtained as the Minkowski sum of $(1,0),(2,1),(1,1),(1,2)$, and $(0,1)$. Before the next intersection with a facet of $[0,6]^{3}$ is considered, $\tilde{d}\left(u, F_{2}^{+}\right)$is updated to 5 as $d\left(u, u^{\prime}\right)=2$ and $u_{2}^{\prime}=3$, see Figure 5 where the vertex $u^{\prime}$ is coloured black while $u$ and $v$ are coloured red. The second edge on the path from $u$ to $u^{\prime}$ satisfies $e_{2} \geq 2$. Consequently, $g_{2}^{+}$is updated to $g+\widetilde{d}\left(u, F_{2}^{+}\right)+\widetilde{d}\left(v, F_{2}^{+}\right)-6=1+5+0-6=0$. Thus, $\delta\left(F_{2}^{+}\right)=6$ which is impossible since $\bar{v}_{2}=(6,6) \notin \mathscr{V}_{2,6,0}$; that is, there is no shelling with the current choice of $F_{1}^{-}$. The same holds for any choice of $F_{1}^{-}$since any lattice $(2,6)$-polytope of diameter at least 5 includes at least one edge $e$ such that $\|e\|_{\infty} \geq 2$. Consequently, there is no shelling for $\{u, v\}=\{(0,0,0),(6,6,6)\}$. Table 2 lists the 69 considered pairs $\{u, v\}$ of vertices of a lattice $(3,6)$-polytope $P$ such that $d(u, v)=11$ where $P$ is assumed to have a nonempty intersection with each facet of $[0,6]^{3}$.

\begin{tabular}{c|c}
$u$ & $v$ \\
\hline$(0,0,0)$ & $(6,6,6)$ \\
$(0,0,1)$ & $(5,5,4),(5,5,5),(5,5,6),(5,6,4),(5,6,5),(5,6,6),(6,6,4),(6,6,5)$ \\
$(0,0,2)$ & $(5,5,3),(5,5,4),(5,5,5),(5,6,3),(5,6,4),(5,6,5),(6,6,3),(6,6,4)$ \\
$(0,0,3)$ & $(5,5,2),(5,5,3),(5,5,4),(5,6,2),(5,6,3),(5,6,4),(6,6,2),(6,6,3)$ \\
$(0,1,1)$ & $(5,4,4),(5,4,5),(5,4,6),(5,5,5),(5,5,6),(6,4,4),(6,4,5),(6,5,5)$ \\
$(0,1,2)$ & $(5,4,3),(5,4,4),(5,4,5),(5,5,3),(5,5,4),(5,5,5),(5,6,4),(6,4,3),(6,4,4),(6,4,5),(6,5,3),(6,5,4)$ \\
$(0,1,3)$ & $(6,4,2),(6,4,3),(6,4,4),(6,5,2),(6,5,3),(6,6,2)$ \\
$(0,2,2)$ & $(6,3,4),(6,4,4)$ \\
$(0,2,3)$ & $(6,3,2),(6,3,4),(6,4,2),(6,4,3),(6,5,2)$ \\
$(1,1,1)$ & $(4,5,5),(5,5,5)$ \\
$(1,1,2)$ & $(5,5,3),(5,5,4)$ \\
$(1,1,3)$ & $(5,5,2),(5,5,3),(5,6,2),(6,6,2)$ \\
$(1,2,2)$ & $(5,4,4)$ \\
$(1,2,3)$ & $(6,5,2)$ \\
$(2,2,3)$ & $(4,5,2)$ \\
\hline
\end{tabular}

Table 2 All considered pairs $\{u, v\}$ for $(d, k, g)=(3,6,1)$ 
4.2 Determination of $\delta(5,3)$

The determination of $\delta(5,3)$ requires the list of all lattice $(4,3)$-polytopes of diameter 8 up to the symmetries of $[0,3]^{4}$. In order to obtain all lattice $(4,3)$-polytopes of diameter 8 , we first determine all lattice $(4,3)$-polytopes of diameter 8 with a nonempty intersection with each facet of $[0,3]^{4}$ by running the algorithm for $(d, k, g)=(4,3,1)$. Then, using the procedure described in Section 3.3 we can use the output of the algorithm for $(d, k, g)=(4,3,1)$ to determine all the lattice $(4,3)$-polytopes of diameter 8 with an empty intersection with at least one facet of $[0,3]^{4}$. Note that running the algorithm for $(d, k, g)=(4,3,1)$ requires the list of all the lattice $(3,3)$-polytopes of diameter 5 or 6 . This is achieved by running the algorithm for $(d, k, g)=(3,3,2)$ and using the procedure described in Section 3.3

Table 3 lists the 6 considered pairs $\{u, v\}$ of vertices of a lattice $(3,3)$-polytope $P$ such that $d(u, v)=6$ where $P$ is assumed to have a non-empty intersection with each facet of $[0,3]^{3}$.

\begin{tabular}{c|c}
$u$ & $v$ \\
\hline$(0,0,0)$ & $(3,3,3)$ \\
$(0,0,1)$ & $(2,3,2),(2,3,3),(3,3,1),(3,3,2)$ \\
$(0,1,1)$ & $(3,2,2)$
\end{tabular}

Table 3 All considered pairs $\{u, v\}$ for $(d, k, g)=(3,3,1)$

The output of the algorithm is made up of the 9 lattice (3,3)-polytopes of diameter 6 , shown in Figure 6 up to the symmetries of $[0,3]^{3}$. In this figure, the edges of the intersections with the facets of $[0,3]^{3}$ are shown in blue. Using the procedure described in Section 3.3 , one can check that there is no lattice $(3,3)$-polytope of diameter 6 with an empty intersection with at least one facet of $[0,3]^{3}$. In other words, any lattice $(3,3)$-polytope of diameter 6 is, up to the symmetries of $[0,3]^{3}$, one of the 9 polytopes depicted in Figure 6 Table 4 provides the numbers $f_{0}(P)$ and $f_{2}(P)$ of vertices and facets of the 9 polytopes represented in Figure 6 The breakdown by incidence is also indicated. For example, $24\{3\}$ and $8\{3\}+$ $6\{8\}$ indicates that the truncated cube $P_{4}$ has 24 vertices, all belonging to 3 facets, and 14 facets consisting in 8 triangles and 6 octagons.

\begin{tabular}{c|cccc} 
Polytope & $f_{0}(P)$ & Vertex incidence & $f_{2}(P)$ & Facet incidence \\
\hline$P_{1}$ & 26 & $20\{3\}+6\{4\}$ & 18 & $12\{4\}+6\{6\}$ \\
$P_{2}$ & 23 & $20\{3\}+3\{4\}$ & 14 & $9\{4\}+5\{6\}$ \\
$P_{3}$ & 20 & $20\{3\}$ & 12 & $6\{4\}+6\{6\}$ \\
$P_{4}$ & 24 & $24\{3\}$ & 14 & $8\{3\}+6\{8\}$ \\
$P_{5}$ & 24 & $24\{3\}$ & 14 & $4\{3\}+3\{4\}+4\{6\}+3\{8\}$ \\
$P_{6}$ & 23 & $22\{3\}+1\{4\}$ & 14 & $4\{3\}+3\{4\}+4\{6\}+2\{7\}+1\{8\}$ \\
$P_{7}$ & 23 & $22\{3\}+1\{4\}$ & 14 & $4\{3\}+3\{4\}+4\{6\}+2\{7\}+1\{8\}$ \\
$P_{8}$ & 22 & $22\{3\}$ & 13 & $2\{3\}+4\{4\}+6\{6\}+1\{8\}$ \\
$P_{9}$ & 24 & $24\{3\}$ & 14 & $6\{4\}+8\{6\}$
\end{tabular}

Table 4 Some combinatorial properties of the lattice (3,3)-polytopes with maximal diameter. 

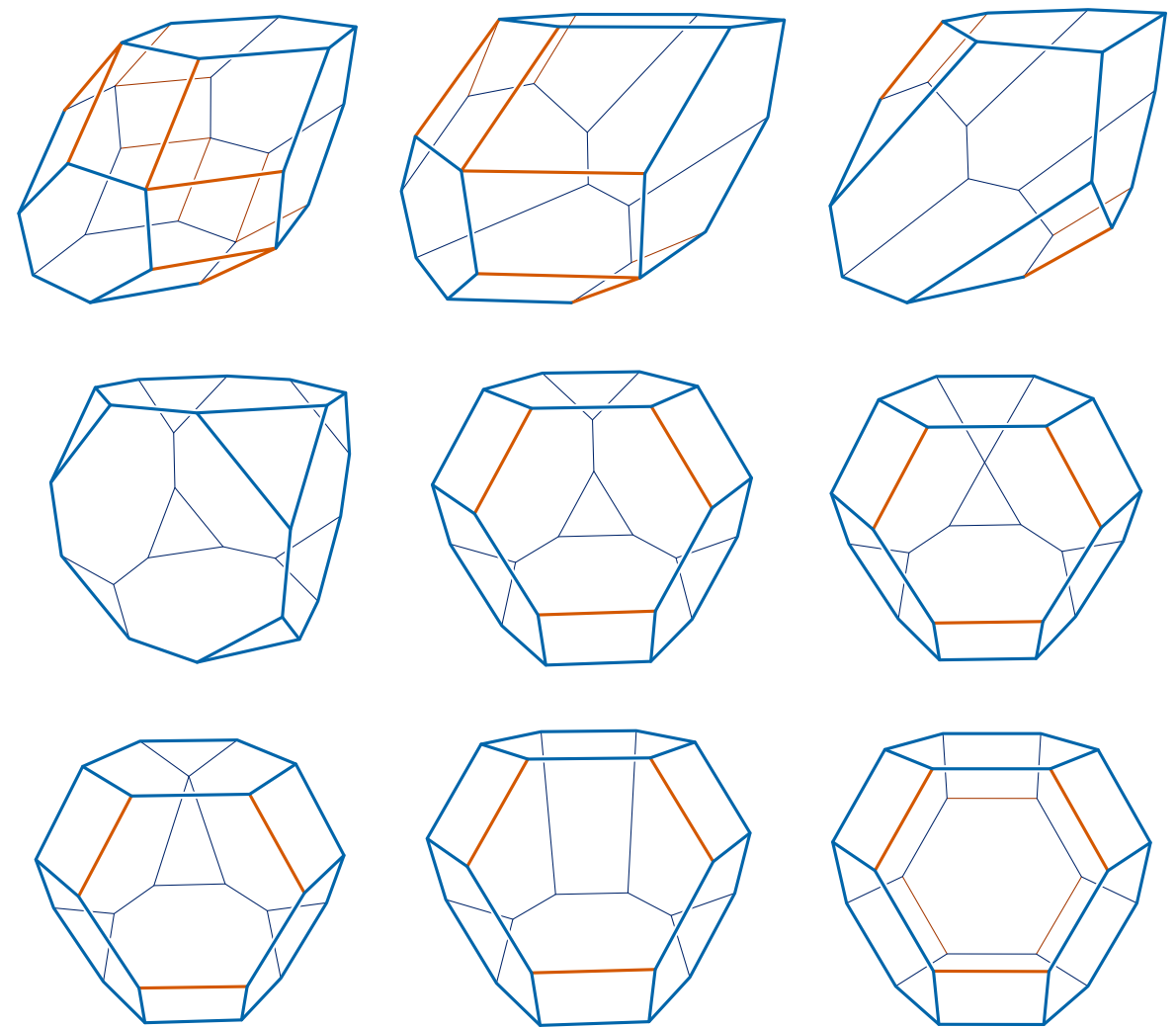

Fig. 6 All, up to the symmetries of $[0,3]^{3}$, lattice $(3,3)$-polytopes of diameter 6

Acknowledgements The authors thank the anonymous referees for their valuable comments. This work was partially supported by the Natural Sciences and Engineering Research Council of Canada Discovery Grant program (RGPIN-2015-06163), by the Exceptional Opportunity program (ESROP), and by the ANR project SoS (Structures on Surfaces) ANR-17-CE40-0033.

\section{References}

1. Acketa, D., Žunić, J.: On the maximal number of edges of convex digital polygons included into an $m \times m$-grid. Journal of Combinatorial Theory A 69, 358-368 (1995)

2. Balog, A., Bárány, I.: On the convex hull of the integer points in a disc. In: Proceedings of the Seventh Annual Symposium on Computational Geometry, pp. 162-165 (1991)

3. Barnette, D.: An upper bound for the diameter of a polytope. Discrete Mathematics 10, 9-13 (1974)

4. Chadder, N., Deza, A.: Computational determination of the largest lattice polytope diameter. In: Proceedings of the IX Latin and American Algorithms, Graphs and Optimization Symposium, Electronic Notes in Discrete Mathematics, vol. 62, pp. 105-110 (2017)

5. Del Pia, A., Michini, C.: On the diameter of lattice polytopes. Discrete and Computational Geometry 55, 681-687 (2016)

6. Deza, A., Manoussakis, G., Onn, S.: Primitive zonotopes. Discrete and Computational Geometry 60, 27-39 (2018)

7. Deza, A., Pournin, L.: Improved bounds on the diameter of lattice polytopes. Acta Mathematica Hungarica 154, 457-469 (2018) 
8. Deza, M., Onn, S.: Lattice-free polytopes and their diameter. Discrete and Computational Geometry 13, 59-75 (1995)

9. Eisenbrand, F., Hähnle, N., Razborov, A., Rothvoß, T.: Diameter of polyhedra: limits of abstraction. Mathematics of Operations Research 35, 786794 (2010)

10. Fukuda, K.: Lecture notes: Polyhedral computation. http://www-oldurls.inf.ethz.ch/ personal/fukudak/lect/pclect/notes2015/

11. Kalai, G., Kleitman, D.: A quasi-polynomial bound for the diameter of graphs of polyhedra. Bulletin of the American Mathematical Society 26, 315-316 (1992)

12. Klee, V.K., Walkup, D.: The $d$-step conjecture for polyhedra of dimension $d<6$. Acta Mathematica 117, 53-78 (1967)

13. Kleinschmidt, P., Onn, S.: On the diameter of convex polytopes. Discrete Mathematics 102, 75-77 (1992)

14. Labbé, J.P., Manneville, T., Santos, F.: Hirsch polytopes with exponentially long combinatorial segments. Mathematical Programming 165, 663-688 (2017)

15. Larman, D.: Paths on polytopes. Proceedings of the London Mathematical Society 20, 161-178 (1970)

16. Naddef, D.: The Hirsch conjecture is true for (0,1)-polytopes. Mathematical Programming 45, 109-110 (1989)

17. Santos, F.: A counterexample to the Hirsch conjecture. Annals of Mathematics 176, 383-412 (2012)

18. Sukegawa, N.: Improving bounds on the diameter of a polyhedron in high dimensions. Discrete Mathematics 340, 2134-2142 (2017)

19. Thiele, T.: Extremalprobleme für Punktmengen. Diplomarbeit, Freie Universität Berlin (1991)

20. Todd, M.: An improved Kalai-Kleitman bound for the diameter of a polyhedron. SIAM Journal on Discrete Mathematics 28, 1944-1947 (2014)

21. Ziegler, G.: Lectures on Polytopes. Graduate Texts in Mathematics. Springer (1995) 